\title{
LOWERING TOPOLOGICAL ENTROPY OVER SUBSETS REVISITED
}

\author{
WEN HUANG, XIANGDONG YE, AND GUOHUA ZHANG
}

\begin{abstract}
Let $(X, T)$ be a topological dynamical system. Denote by $h(T, K)$ and $h^{B}(T, K)$ the covering entropy and dimensional entropy of $K \subseteq X$, respectively. $(X, T)$ is called D-lowerable (resp. lowerable) if for each $0 \leq h \leq$ $h(T, X)$ there is a subset (resp. closed subset) $K_{h}$ with $h^{B}\left(T, K_{h}\right)=h$ (resp. $\left.h\left(T, K_{h}\right)=h\right)$ and is called D-hereditarily lowerable (resp. hereditarily lowerable) if each Souslin subset (resp. closed subset) is D-lowerable (resp. lowerable).

In this paper it is proved that each topological dynamical system is not only lowerable but also D-lowerable, and each asymptotically $h$-expansive system is D-hereditarily lowerable. A minimal system which is lowerable and not hereditarily lowerable is demonstrated.
\end{abstract}

\section{INTRODUCTION}

This paper is a continuation of the research done in [17] by the same authors.

Throughout the paper, by a topological dynamical system (t.d.s.) $(X, T)$ we mean a compact metric space $X$ together with a homeomorphism $T: X \rightarrow X$. Let $(X, T)$ be a t.d.s. and $K \subseteq X$. Denote by $h(T, K)$ and $h^{B}(T, K)$ the covering entropy and dimensional entropy of $K \subseteq X$ introduced in [2] and [4] respectively. Motivated by [24 26, 36], in [17] the authors studied the question whether for each $0 \leq h \leq$ $h(T, X)$ there is a closed subset of $X$ with entropy $h$. Inspired by [40, Remark 5.13], in [17] we call $(X, T)$

(1) lowerable if for each $0 \leq h \leq h(T, X)$ there is a closed $K \subseteq X$ with $h(T, K)=h$;

(2) hereditarily lowerable if each closed subset is lowerable; i.e. for each closed $K \subseteq X$ and any $0 \leq h \leq h(T, K)$ there is a closed $K_{h} \subseteq K$ with $h(T, K)=$ $h$

(3) hereditarily uniformly lowerable if for each closed subset $K \subseteq X$ and any $0 \leq h \leq h(T, K)$ there is a closed $K_{h} \subseteq K$ such that $h\left(T, K_{h}\right)=h$ and $K_{h}$ has at most one limit point.

Then the question is divided further into the following questions in [17:

Question 1. Is any t.d.s. lowerable?

Question 2. Is any t.d.s. hereditarily lowerable?

Received by the editors July 11, 2012 and, in revised form, December 4, 2012. 2010 Mathematics Subject Classification. Primary 37B40, 37A35, 37B10, 37A05.

Key words and phrases. Entropy, principal extension, lowerable, hereditarily lowerable.

The first author was supported by NNSF of China (11225105), the Fok Ying Tung Education Foundation and the Fundamental Research Funds for the Central Universities.

The first and second authors were supported by NNSF of China (11071231).

The third author was supported by FANEDD (201018) and NSFC (11271078). 
Question 3. Is any t.d.s. hereditarily uniformly lowerable?

We remark that the reason we ask Question 3 in such a way is that in 40 the authors showed that if $(X, T)$ is a t.d.s. and $K \subset X$ is a compact infinite subset, then there is a countable subset $K^{\prime} \subset K$ (the derived set of which has at most one limit point) with $h\left(T, K^{\prime}\right)=h(T, K)$. In [17] the authors showed that each t.d.s. with finite entropy is lowerable, and that a t.d.s. is hereditarily uniformly lowerable iff it is asymptotically $h$-expansive. In particular, each hereditarily uniformly lowerable t.d.s. has finite entropy. Moreover, a principal extension preserves the lowerable, hereditarily lowerable and hereditarily uniformly lowerable properties. Though we completely answered Question 3. but Question 1 in the case $h(T, X)=+\infty$ and Question 2 still remain open in [17.

Let $X$ be a metric space; the Souslin sets are the sets of the form

$$
E=\bigcup_{i_{1} \in \mathbb{N}, i_{2} \in \mathbb{N}, \cdots} \bigcap_{k \in \mathbb{N}} E_{i_{1}, \cdots, i_{k}},
$$

where $E_{i_{1}, \cdots, i_{k}}$ is a closed set for each finite sequence $\left\{i_{1}, \cdots, i_{k}\right\}$ of positive integers. Observe that each Borel set is Souslin, the pre-image of a Souslin set under a continuous map is Souslin, and if the underlying metric spaces are complete, then any continuous image of a Souslin set is Souslin. The well-known result in fractal geometry [10,27] states that (for the definition of Hausdorff dimension see [10,27])

Proposition 1.1. Let $K \subseteq \mathbb{R}^{n}$ be a non-empty Souslin subset. Then for each $0 \leq h<\operatorname{dim}_{H}(K)$ there is a compact subset $K_{h}$ of $K$ with $\operatorname{dim}_{H}\left(K_{h}\right)=h$, where $\operatorname{dim}_{H}(*)$ denotes the Hausdorff dimension of a subset $*$ in $\mathbb{R}^{n}$.

Inspired by this, for a t.d.s. $(X, T)$ we call it

(1) D-lowerable if for each $0 \leq h \leq h(T, X)$ there is a subset $K_{h}$ with $h^{B}\left(T, K_{h}\right)$ $=h$;

(2) D-hereditarily lowerable if each Souslin subset is D-lowerable; i.e. for each Souslin set $K \subseteq X$ and any $0 \leq h \leq h^{B}(T, K)$ there is $K_{h} \subseteq K$ with $h^{B}\left(T, K_{h}\right)=h$.

Thus, we have two other additional questions:

Question 4. Is any t.d.s. D-lowerable?

Question 5. Is any t.d.s. D-hereditarily lowerable?

We emphasize that, in fact, [17, Theorem 4.4] tells us that each t.d.s. with finite entropy is D-lowerable.

In this paper, we get complete answers to Questions 1 1 and 4, and partial answers to Questions 2 and 5 (Question 3 was answered by [17, Theorem 7.7]). Namely, with the help of a relative version of the well-known Sinai Theorem we prove that each t.d.s. is not only lowerable but also D-lowerable. We shall construct a minimal lowerable t.d.s. which is not hereditarily lowerable. Moreover, we also prove that each asymptotically $h$-expansive t.d.s. is D-hereditarily lowerable. However, there remain some unsolved interesting questions. For example, is there a lowerable t.d.s. with finite entropy which is not hereditarily lowerable?

The paper is organized as follows. In Section 2 the definitions of cover entropy and dimensional entropy of subsets are introduced. In Section 3, a minimal lowerable t.d.s. which is not hereditarily lowerable is presented. Then in Section 4 it is proved that each t.d.s. is not only lowerable but also D-lowerable with the help of 
a relative version of the well-known Sinai Theorem. In the last section, it is shown that each asymptotically $h$-expansive t.d.s. is D-hereditarily lowerable.

\section{PRELIMINARY}

Let $(X, T)$ be a t.d.s., $K \subseteq X$ and $\mathcal{W}$ a collection of subsets of $X$. We shall write $K \succeq \mathcal{W}$ if $K \subseteq W$ for some $W \in \mathcal{W}$; otherwise $K \nsucceq \mathcal{W}$. If $\mathcal{W}_{1}$ is another family of subsets of $X, \mathcal{W}$ is said to be finer than $\mathcal{W}_{1}$ (we shall write $\mathcal{W} \succeq \mathcal{W}_{1}$ ) when $W \succeq \mathcal{W}_{1}$ for each $W \in \mathcal{W}$. We shall say that a numerical function increases (resp. decreases) with respect to (w.r.t.) a set variable $K$ or a family variable $\mathcal{W}$ if the value never decreases (resp. increases) when $K$ is replaced by a set $K_{1}$ with $K_{1} \subseteq K$ or when $\mathcal{W}$ is replaced by a family $\mathcal{W}_{1}$ with $\mathcal{W}_{1} \succeq \mathcal{W}$.

By a cover of $X$ we mean a finite family of Borel subsets with union $X$, and by a partition a cover whose elements are disjoint. Denote by $\mathcal{C}_{X}\left(\right.$ resp. $\left.\mathcal{C}_{X}^{o}, \mathcal{P}_{X}\right)$ the set of covers (resp. open covers, partitions). If $\alpha \in \mathcal{P}_{X}$ and $x \in X$, then let $\alpha(x)$ be the element of $\alpha$ containing $x$.

Given $\mathcal{U}_{1}, \mathcal{U}_{2} \in \mathcal{C}_{X}$, set $\mathcal{U}_{1} \vee \mathcal{U}_{2}=\left\{U_{1} \cap U_{2}: U_{1} \in \mathcal{U}_{1}, U_{2} \in \mathcal{U}_{2}\right\}$; obviously $\mathcal{U}_{1} \vee \mathcal{U}_{2} \in \mathcal{C}_{X}$ and $\mathcal{U}_{1} \vee \mathcal{U}_{2} \succeq \mathcal{U}_{1} . \mathcal{U}_{1} \succeq \mathcal{U}_{2}$ need not imply that $\mathcal{U}_{1} \vee \mathcal{U}_{2}=\mathcal{U}_{1}$, $\mathcal{U}_{1} \succeq \mathcal{U}_{2}$ iff $\mathcal{U}_{1}$ is equivalent to $\mathcal{U}_{1} \vee \mathcal{U}_{2}$ in the sense that each refines the other. For each $\mathcal{U} \in \mathcal{C}_{X}$ and any $m, n \in \mathbb{Z}_{+}$with $m \leq n$ we set $\mathcal{U}_{m}^{n}=\bigvee_{i=m}^{n} T^{-i} \mathcal{U}$. Moreover, if $(X, T)$ is a t.d.s., then let $\operatorname{diam}(K)$ be the diameter of $K$ and put $\|\mathcal{W}\|=\sup \{\operatorname{diam}(W): W \in \mathcal{W}\}$. Thus if $\mathcal{U} \in \mathcal{C}_{X}^{o}$, then $\mathcal{U}$ has a Lebesgue number $\lambda>0$, and so $\mathcal{W} \succeq \mathcal{U}$ when $\|\mathcal{W}\|<\lambda$.

2.1. Covering entropy of subsets. Let $(X, T)$ be a t.d.s., and let $K \subseteq X$ and $\mathcal{U} \in \mathcal{C}_{X}$. Set $N(\mathcal{U}, K)$ to be the minimal cardinality of sub-families $\mathcal{V} \subseteq \mathcal{U}$ with $\cup \mathcal{V} \supseteq K$, where $\cup \mathcal{V}=\bigcup_{V \in \mathcal{V}} V$. We write $N(\mathcal{U}, \emptyset)=1$ by convention. Obviously, $N(\mathcal{U}, T(K))=N\left(T^{-1} \mathcal{U}, K\right)$. Let

$$
h_{\mathcal{U}}(T, K)=\limsup _{n \rightarrow+\infty} \frac{1}{n} \log N\left(\mathcal{U}_{0}^{n-1}, K\right) .
$$

Clearly $h_{\mathcal{U}}(T, K)$ increases w.r.t. $\mathcal{U}$. Define the covering entropy of $K$ by

$$
h(T, K)=\sup _{\mathcal{U} \in \mathcal{C}_{X}^{o}} h_{\mathcal{U}}(T, K),
$$

and define the topological entropy of $(X, T)$ by $h_{\text {top }}(T, X)=h(T, X)$.

Let $(X, T)$ and $(Y, S)$ be t.d.s.s. We say that $\pi:(X, T) \rightarrow(Y, S)$ is a factor map if $\pi$ is a continuous surjection and $\pi \circ T=S \circ \pi$. It is easy to check that

Proposition 2.1. Let $(X, T)$ and $(Y, S)$ be t.d.s.s. Then

(1) $h(T, K) \geq h(S, \pi(K))$ if $\pi:(X, T) \rightarrow(Y, S)$ is a factor map and $K \subseteq X$;

(2) $h(T \times S, X \times Y)=h(T, X)+h(S, Y)$.

We may also obtain the cover entropy of subsets using Bowen's separated and spanning sets (see [38, $\left.\mathrm{P}_{168-174}\right]$ ). Let $(X, T)$ be a t.d.s. with $d$ a compatible metric on $X$. For each $n \in \mathbb{N}$ we define a new metric $d_{n}$ on $X$ by

$$
d_{n}(x, y)=\max _{0 \leq i \leq n-1} d\left(T^{i} x, T^{i} y\right) .
$$

Let $\epsilon>0$ and $K \subseteq X$. A subset $F$ of $X$ is said to $(n, \epsilon)$-span $K$ w.r.t. $T$ if for each $x \in K$, there is $y \in F$ with $d_{n}(x, y) \leq \epsilon$; a subset $E$ of $K$ is said to be $(n, \epsilon)$ separated w.r.t. $T$ if $x, y \in E, x \neq y$ implies $d_{n}(x, y)>\epsilon$. Let $r_{n}(d, T, \epsilon, K)$ denote 
the smallest cardinality of any $(n, \epsilon)$-spanning set for $K$ w.r.t. $T$ and $s_{n}(d, T, \epsilon, K)$ denote the largest cardinality of any $(n, \epsilon)$-separated subset of $K$ w.r.t. $T$. We write $r_{n}(d, T, \epsilon, \emptyset)=s_{n}(d, T, \epsilon, \emptyset)=1$ by convention. Put

$$
r(d, T, \epsilon, K)=\limsup _{n \rightarrow+\infty} \frac{1}{n} \log r_{n}(d, T, \epsilon, K)
$$

and

Then put

$$
s(d, T, \epsilon, K)=\limsup _{n \rightarrow+\infty} \frac{1}{n} \log s_{n}(d, T, \epsilon, K) .
$$

$$
h_{*}(d, T, K)=\lim _{\epsilon \rightarrow 0+} r(d, T, \epsilon, K) \text { and } h^{*}(d, T, K)=\lim _{\epsilon \rightarrow 0+} s(d, T, \epsilon, K) .
$$

It is well known that $h_{*}(d, T, K)=h^{*}(d, T, K)$ is independent of the choice of a compatible metric $d$ on the space $X$. Now, if $\mathcal{U} \in \mathcal{C}_{X}^{o}$ has a Lebesgue number $\delta>0$, then, for any $\delta^{\prime} \in\left(0, \frac{\delta}{2}\right)$ and each $\mathcal{V} \in \mathcal{C}_{X}^{o}$ with $\|\mathcal{V}\| \leq \delta^{\prime}$, one has

$$
N\left(\mathcal{U}_{0}^{n-1}, K\right) \leq r_{n}\left(d, T, \delta^{\prime}, K\right) \leq s_{n}\left(d, T, \delta^{\prime}, K\right) \leq N\left(\mathcal{V}_{0}^{n-1}, K\right)
$$

for each $n \in \mathbb{N}$. So if $\left\{\mathcal{U}_{n}\right\}_{n \in \mathbb{N}} \subseteq \mathcal{C}_{X}^{o}$ satisfies $\left\|\mathcal{U}_{n}\right\| \rightarrow 0$ as $n \rightarrow+\infty$, then

$$
h_{*}(d, T, K)=h^{*}(d, T, K)=\lim _{n \rightarrow+\infty} h_{\mathcal{U}_{n}}(T, K)=h(T, K) .
$$

In this case, it is obvious that $h(T, \bar{K})=h(T, K)$.

2.2. Dimensional entropy of subsets. Now we recall the concept of dimensional entropy introduced and studied in 4 .

Let $(X, T)$ be a t.d.s. and $\mathcal{U} \in \mathcal{C}_{X}$. For $K \subseteq X$ let

$$
n_{T, \mathcal{U}}(K)= \begin{cases}0, & \text { if } K \nsucceq \mathcal{U}, \\ +\infty, & \text { if } T^{i} K \succeq \mathcal{U} \text { when } i \in \mathbb{Z}_{+}, \\ k, & k=\max \left\{j \in \mathbb{N}: T^{i}(K) \succeq \mathcal{U} \text { when } 0 \leq i \leq j-1\right\} .\end{cases}
$$

For $k \in \mathbb{N}$, we define $\mathfrak{C}(T, \mathcal{U}, K, k)$ to be the family of all $\mathcal{E}$, where $\mathcal{E}$ is a countable family of subsets of $X$ such that $K \subseteq \cup \mathcal{E}$ and $\mathcal{E} \succeq \mathcal{U}_{0}^{k-1}$. Then for each $\lambda \in \mathbb{R}$ set

$$
m_{T, \mathcal{U}}(K, \lambda, k)=\inf _{\mathcal{E} \in \mathfrak{C}(T, \mathcal{U}, K, k)} m(T, \mathcal{U}, \mathcal{E}, \lambda),
$$

where

$$
m(T, \mathcal{U}, \mathcal{E}, \lambda)=\sum_{E \in \mathcal{E}} e^{-\lambda n_{T, \mathcal{U}}(E)} .
$$

Here, by convention: $0 \cdot \infty=0$ and $m_{T, \mathcal{U}}(\emptyset, \lambda, k)=+\infty$ if $\lambda<0 ; 1$ if $\lambda=0$; 0 if $\lambda>0$. As $m_{T, \mathcal{U}}(K, \lambda, k)$ is increasing w.r.t. $k$, we can define

$$
m_{T, \mathcal{U}}(K, \lambda)=\lim _{k \rightarrow+\infty} m_{T, \mathcal{U}}(K, \lambda, k) .
$$

Notice that $m_{T, \mathcal{U}}(K, \lambda) \leq m_{T, \mathcal{U}}\left(K, \lambda^{\prime}\right)$ for $\lambda \geq \lambda^{\prime}$ and $m_{T, \mathcal{U}}(K, \lambda) \notin\{0,+\infty\}$ for at most one $\lambda[4$. We define the dimensional entropy of $K$ relative to $\mathcal{U}$ by

$$
h_{\mathcal{U}}^{B}(T, K)=\inf \left\{\lambda \in \mathbb{R}: m_{T, \mathcal{U}}(K, \lambda)=0\right\}=\sup \left\{\lambda \in \mathbb{R}: m_{T, \mathcal{U}}(K, \lambda)=+\infty\right\} .
$$

The dimensional entropy of $K$ is defined by

$$
h^{B}(T, K)=\sup _{\mathcal{U} \in \mathcal{C}_{X}^{o}} h_{\mathcal{U}}^{B}(T, K) .
$$

Note that $h_{\mathcal{U}}^{B}(T, K)$ increases w.r.t. $\mathcal{U} \in \mathcal{C}_{X}$. Thus if $(X, T)$ is a t.d.s. and $\left\{\mathcal{U}_{n}\right\}_{n \in \mathbb{N}}$ $\subseteq \mathcal{C}_{X}^{o}$ satisfies $\left\|\mathcal{U}_{n}\right\| \rightarrow 0$ as $n \rightarrow+\infty$, then $\lim _{n \rightarrow+\infty} h_{\mathcal{U}_{n}}^{B}(T, K)=h^{B}(T, K)$. 
The following result is basic (see 4, Propositions 1 and 2] or [17, Proposition $2.3])$.

Proposition 2.2. Let $(X, T)$ be a t.d.s., $K_{1}, K_{2}, \cdots, K \subseteq X$ and $\mathcal{U} \in \mathcal{C}_{X}$. Then

(1) $h_{\mathcal{U}}(T, X)=h_{\mathcal{U}}^{B}(T, X)$ if $\mathcal{U} \in \mathcal{C}_{X}^{o}$, so $h(T, X)=h^{B}(T, X)$;

(2) $h_{\mathcal{U}}^{B}\left(T, \bigcup_{n \in \mathbb{N}} K_{n}\right)=\sup _{n \in \mathbb{N}} h_{\mathcal{U}}^{B}\left(T, K_{n}\right)$, so

$$
h^{B}\left(T, \bigcup_{n \in \mathbb{N}} K_{n}\right)=\sup _{n \in \mathbb{N}} h^{B}\left(T, K_{n}\right) ;
$$

(3) for each $m \in \mathbb{N}$ and $i \geq 0, h_{T^{-i} \mathcal{U}}^{B}\left(T^{m}, K\right)=h_{\mathcal{U}}^{B}\left(T^{m}, T^{i} K\right)$, so $h^{B}\left(T^{m}, K\right)=$ $h^{B}\left(T^{m}, T^{i} K\right)$

(4) for each $m \in \mathbb{N}, h_{\mathcal{U}_{0}^{m-1}}^{B}\left(T^{m}, K\right)=m h_{\mathcal{U}}^{B}(T, K)$, so $h^{B}\left(T^{m}, K\right)=m h^{B}(T, K)$.

Thus, by Proposition $2.2(2), h^{B}(T, E)$ increases w.r.t. $E \subseteq X$, and if $E \subseteq X$ is a non-empty countable set, then $h^{B}(T, E)=0$. It is worth mentioning that

(1) $h_{\mathcal{U}}^{B}(T, \emptyset)=h_{\mathcal{U}}(T, \emptyset)=0$ for any $\mathcal{U} \in \mathcal{C}_{X}$, and so $h^{B}(T, \emptyset)=h(T, \emptyset)=0$;

(2) when $\emptyset \neq K \subseteq X$, one has $h_{\mathcal{U}}(T, K) \geq h_{\mathcal{U}}^{B}(T, K) \geq 0$ for any $\mathcal{U} \in \mathcal{C}_{X}$, and so

$$
h(T, K) \geq h^{B}(T, K) \geq 0 .
$$

2.3. Hausdorff dimension and dimensional entropy. Let $(X, d)$ be a metric space. We first recall the definition of a Hausdorff dimension of a subset $A \subset X$. Fix $t \geq 0$. For each $\delta>0$ and subset $A \subset X$, we define

$$
H_{d}^{t, \delta}(A)=\inf \left\{\sum_{i=1}^{+\infty} \operatorname{diam}\left(U_{i}\right)^{t}\right\},
$$

where the infimum is taken over all countable covers $\left\{U_{i}: i=1,2, \cdots\right\}$ of $A$ of diameter not exceeding $\delta$. Since $H_{d}^{t, \delta}(A)$ increases as $\delta$ decreases for any $A \subseteq X$, we can define

$$
H_{d}^{t}(A)=\lim _{\delta \rightarrow 0} H_{d}^{t, \delta}(A)=\sup _{\delta>0} H_{d}^{t, \delta}(A) .
$$

The case $H_{d}^{t}(A)=+\infty$ is not excluded. Fix $A \subseteq X$. Since for every $0<\delta \leq 1$ the function $t \mapsto H_{d}^{t, \delta}(A)$ is non-increasing, so is the function $t \mapsto H_{d}^{t}(A)$. Moreover, if $0<s<t$, then for every $\delta>0$

$$
H_{d}^{s, \delta}(A) \geq \delta^{s-t} H_{d}^{t, \delta}(A),
$$

which implies that if $H_{d}^{t}(A)>0$, then $H_{d}^{s}(A)=+\infty$. Thus there is a unique value $H_{d}(A) \in[0,+\infty]$, which is called the Hausdorff dimension of $A$ with respect to the metric $d$ on $X$, such that

$$
H_{d}^{t}(A)= \begin{cases}+\infty, & \text { if } 0 \leq t<H_{d}(A), \\ 0, & \text { if } H_{d}(A)<t<\infty .\end{cases}
$$

The Hausdorff dimension is a monotone function of sets, i.e. if $A \subseteq B$, then $H_{d}(A) \leq H_{d}(B)$. Moreover if $\left\{A_{n}\right\}_{n \geq 1}$ is a countable family of subsets of $X$, then

$$
H_{d}\left(\bigcup_{n=1}^{\infty} A_{n}\right)=\sup _{n \geq 1} H_{d}\left(A_{n}\right) .
$$

Hence if $E \subset X$ is countable, then $H_{d}(E)=0$. 
In the following we investigate the interrelation of Hausdorff dimension and dimensional entropy of a set in some specific t.d.s. Let $(X, T)$ be a t.d.s. with metric $d$. We assume that $T$ is Lipschitz continuous with the Lipschitz constant L, i.e. $d(T x, T y) \leq L d(x, y)$ for any $x, y \in X$.

The following result is just [30, Theorem 1].

Lemma 2.3. Let $(X, T)$ be a Lipschitz continuous t.d.s. with Lipschitz constant $L>1$ associated to the metric d. Then

$$
H_{d}(C) \geq \frac{h^{B}(T, C)}{\log L}
$$

for any subset $C \subseteq X$.

The following result is [11, Lemma 5.4].

Lemma 2.4. Let $(X, T)$ be a t.d.s. with metric $d$. If there exist $\epsilon>0$ and $L>1$ such that $d(T x, T y) \geq L d(x, y)$ whenever $d(x, y)<\epsilon$, then

$$
H_{d}(C) \leq \frac{h^{B}(T, C)}{\log L}
$$

for any subset $C \subseteq X$.

Proposition 2.5. Let $\mathbb{T}=\mathbb{R} / \mathbb{Z}$ be the unit circle of a complex plane with the metric

$$
d\left(e^{2 \pi i x}, e^{2 \pi i y}\right)=\inf _{k \in \mathbb{Z}}|x-y-k|
$$

for any $x, y \in \mathbb{R}$. For $m \in \mathbb{N}$ with $m \geq 2, T_{m}$ is defined by $T_{m}(z)=z^{m}$ for $z \in \mathbb{T}$. Then

(1) $H_{d}(C)=\frac{h^{B}\left(T_{m}, C\right)}{\log m}$ for any subset $C \subseteq \mathbb{T}$.

(2) $\left(\mathbb{T}, T_{m}\right)$ is D-hereditarily lowerable.

Proof. (1) follows from Lemmas 2.3 and 2.4 by setting $L=m$. Since the metric $d$ and the Euclidean metric on $\mathbb{T}$ are Lipschitz equivalent, (2) follows from (1) and Proposition 1.1 .

Remark 2.6. Proposition 2.5 (1) first appeared in [12, Proposition III.1].

Remark 2.7. Recall that in [40, Remark 5.13] the authors showed that if $(X, T)$ is a t.d.s. and $K \subset X$ is a compact subset, then there is a countable subset $K^{\prime} \subset K$ (the derived set of which has at most one limit point) with $h\left(T, K^{\prime}\right)=h(T, K)$. Weiss showed us a proof that when $(X, T)$ is minimal and $X$ is infinite, then there exists a countable subset $K$ with a unique limit point such that $h(T, K)=h(T, X)$. In fact, this can also be obtained by [40, Theorems 4.2 and 5.7].

\section{Negative answers to Question 2}

In this section, we shall construct a minimal lowerable t.d.s. which is not hereditarily lowerable. First we give a lowerable t.d.s. which is not hereditarily lowerable and then we make it minimal. We remark that the example we get has infinite entropy, and it is not hard to construct an example which has infinite entropy and at the same time is hereditarily lowerable. 
3.1. A general example. First we construct an example (not necessarily minimal) which is lowerable and not hereditarily lowerable. In the next subsection we will modify it such that it is minimal. To do this, we need the following lemma.

Lemma 3.1. Let $\left(X_{i}, T_{i}\right), i \in \mathbb{N}$ be a t.d.s. and $(Y, S)=\prod_{i \in \mathbb{N}}\left(X_{i}, T_{i}\right)$ with $\pi_{i}$ the projection from $Y$ to $X_{i}, i \in \mathbb{N}$. Then

(1) $h\left(T_{1}^{n}, W\right)=n h\left(T_{1}, W\right)$ for each closed $W \subseteq X_{1}$ and any $n \in \mathbb{N}$;

(2) $h\left(T_{j}, \pi_{j} K\right) \leq h(S, K) \leq \sum_{i \in \mathbb{N}} h\left(T_{i}, \pi_{i} K\right)$ for each closed $K \subseteq Y$ and any $j \in \mathbb{N}$.

Proof. (1) It is known; see for example the proof of [38, Theorem 7.10 (i)].

(2) Let $K \subseteq Y$ be closed and $j \in \mathbb{N}$. By Proposition 2.1 (1) clearly $h\left(T_{j}, \pi_{j} K\right) \leq$ $h(S, K)$. For the other direction, without loss of generality we assume $\operatorname{diam}\left(X_{i}\right) \leq 1$ with $d_{i}$ a compatible metric on $X_{i}$ for each $i \in \mathbb{N}$. Let $\rho$ be the metric on $Y$ given by

$$
\rho\left(\left(x_{1}, x_{2}, \cdots\right),\left(y_{1}, y_{2}, \cdots\right)\right)=\sum_{i=1}^{\infty} \frac{d_{i}\left(x_{i}, y_{i}\right)}{2^{i}} .
$$

For each $\epsilon>0$ we can select $N(\epsilon) \in \mathbb{N}$ with $\sum_{i=N(\epsilon)+1}^{\infty} \frac{1}{2^{i}}<\frac{\epsilon}{2}$. Thus

$$
r_{n}(\rho, S, \epsilon, K) \leq \prod_{i=1}^{N(\epsilon)} r_{n}\left(d_{i}, T_{i}, \frac{\epsilon}{2}, \pi_{i} K\right)
$$

which implies

$$
r(\rho, S, \epsilon, K) \leq \sum_{i=1}^{N(\epsilon)} r\left(d_{i}, T_{i}, \frac{\epsilon}{2}, \pi_{i} K\right) \leq \sum_{i \in \mathbb{N}} r\left(d_{i}, T_{i}, \frac{\epsilon}{2}, \pi_{i} K\right),
$$

and so $h(S, K) \leq \sum_{i \in \mathbb{N}} h\left(T_{i}, \pi_{i} K\right)$. This finishes our proof.

Thus, we have

Proposition 3.2. Let $(X, T)$ be a t.d.s. with topological entropy finite but positive. Then $\left(X^{\infty}, S\right)=\prod_{n \in \mathbb{N}}\left(X, T^{n}\right)$ is a lowerable t.d.s. which is not hereditarily lowerable.

Proof. Let $E=\{(x, x, \cdots): x \in X\} \subseteq X^{\infty}$. Now we claim that the subset $E$ in $\left(X^{\infty}, S\right)$ is not lowerable (and so t.d.s. $\left(X^{\infty}, S\right)$ is not hereditarily lowerable) by proving that each closed subset $K$ of $E$ has either infinite topological entropy or zero topological entropy.

Let $\pi_{i}:\left(X^{\infty}, S\right) \rightarrow\left(X, T^{i}\right)$ be the factor map of the $i$-th projection map, $i \in \mathbb{N}$. We shall prove that if $h\left(T, \pi_{1} K\right)>0$, then $h(S, K)=\infty$, and if $h\left(T, \pi_{1} K\right)=0$, then $h(S, K)=0$. In fact, if $h\left(T, \pi_{1} K\right)=0$, then by Lemma 3.1 one has

$$
h(S, K) \leq \sum_{i \in \mathbb{N}} h\left(T^{i}, \pi_{i} K\right)=\sum_{i \in \mathbb{N}} i h\left(T, \pi_{i} K\right)=\sum_{i \in \mathbb{N}} i h\left(T, \pi_{1} K\right)=0 .
$$

Now if $h\left(T, \pi_{1} K\right)>0$, then by Lemma 3.1 one has

$$
h(S, K) \geq h\left(T^{n}, \pi_{n} K\right)=n h\left(T, \pi_{n} K\right)=n h\left(T, \pi_{1} K\right)
$$

for each $n \in \mathbb{N}$, which implies $h(S, K)=\infty$.

Now we shall finish our proof by claiming that $\left(X^{\infty}, S\right)$ is lowerable. In fact, for each $0 \leq h<\infty$ we let $n \in \mathbb{N}$ with $h\left(S_{n}, X^{n}\right)>h$, where $S_{n}=T \times T^{2} \times \cdots \times T^{n}$. 
Note that [17, Theorem 4.4] states that each t.d.s. with finite entropy must be lowerable, whereas, clearly $\left(X^{n}, S_{n}\right)$ is a t.d.s. with finite entropy. Thus there exists a closed $K_{h} \subseteq X^{n}$ with $h\left(S_{n}, K_{h}\right)=h$. Now for each $x_{0} \in X$ we put $K_{h}^{*}=$ $\left\{\left(k_{1}, \cdots, k_{n}, x_{0}, \cdots\right):\left(k_{1}, \cdots, k_{n}\right) \in K_{h}\right\}$. It is easy to check that $h\left(S, K_{h}^{*}\right)=h$; this ends the proof.

We should remark that [17, Theorem 6.1] states that

Let $(X, T)$ be a t.d.s. Then, for any compact $K \subseteq X$ with $h(T, K)>0$,

there is a countable infinite compact subset $K_{\infty} \subseteq K$ with $h\left(T, K_{\infty}\right)=0$, whereas, [40, Theorem 5.9 and Remark 5.13] state that

Let $(X, T)$ be a t.d.s. Then for any compact $K \subseteq X$ there is a countable compact subset $K^{\prime} \subseteq K$ with $h\left(T, K^{\prime}\right)=h(T, K)$.

Thus, the above Proposition 3.2 tells us that these are the best results we may obtain. In view of this, we restate our Question 2 as

Question $2^{\prime}$. Is there a t.d.s. with finite entropy which is not hereditarily lowerable?

It seems to us that a t.d.s. $(X, T)$ is not hereditarily lowerable if it has an ergodic measure with infinite entropy.

3.2. A minimal example. After we finished the construction in the previous subsection, Glasner asked if there is a minimal t.d.s. with the property. We will show that the example in the previous subsection can be made minimal. Recall that a t.d.s. $(Y, T)$ is called proximal orbit dense, or a $P O D$ system, if $(Y, T)$ is totally minimal; and whenever $x, y \in Y$ with $x \neq y$, then for some $n \neq 0, T^{n} y$ is proximal to $x$. An interesting property of POD is that (see [21, Corollary 3.5]):

Proposition 3.3. Let $(X, T)$ be $P O D$ and consider integers $k_{i} \neq 0, i=1, \cdots, n$, with $k_{i} \neq \pm k_{j}$ for $i \neq j$. Then

(1) $\left(X \times \cdots \times X, T^{k_{1}} \times \cdots \times T^{k_{n}}\right)$ is minimal.

(2) The only factors of the flow in (1) are the obvious direct factors.

If, in addition, $(X, T)$ is not isomorphic to $\left(X, T^{-1}\right)$, then (1) and (2) hold for any $k_{i} \neq 0, i=1, \cdots, n$, with $k_{i} \neq k_{j}$ for $i \neq j$.

A special class of POD is

Definition 3.4. A system $(X, T)$ is said to be doubly minimal if for all $x \in X$ and $y \notin\left\{T^{n} x\right\}_{-\infty}^{\infty}$, the orbit of $(x, y)$ is dense in $X \times X$.

The first example of a non-periodic doubly minimal system was constructed in [18] in the symbolic dynamics. Doubly minimal systems are natural in the sense that any ergodic system with zero entropy has a uniquely ergodic model which is doubly minimal [39]. The notion of disjointness between two t.d.s. was introduced in 12, and it is easy to see that two minimal t.d.s. are disjoint iff the product system is minimal [1, Proposition 2.5].

Proposition 3.5. There is a minimal t.d.s. which is not hereditarily lowerable.

Proof. Let $\left(Y_{1}, S_{1}\right)$ be the non-periodic double minimal system constructed in [18]; in particular, $\left(Y_{1}, S_{1}\right)$ is a strictly ergodic t.d.s. with finite entropy. Now let $(Y, S)$ be a minimal t.d.s. with finite positive entropy, which is an extension of $\left(Y_{1}, S_{1}\right)$ with a factor map $\pi$ satisfying that $\left\{y_{1} \in Y_{1}: \pi^{-1}\left(y_{1}\right)\right.$ is a singleton $\}$ is a residual 
subset of $Y_{1}$ (see [8, Theorem 3] for the existence of such a t.d.s. $(Y, S)$, as $\left(Y_{1}, S_{1}\right)$ is a strictly ergodic non-periodic system with finite entropy). Since both $(Y, S)$ and $\left(Y_{1}, S_{1}\right)$ are minimal, it is well known that the factor map $\pi$ is almost 1-1 in the sense that the subset $\left\{y \in Y: \pi^{-1}(\pi y)\right.$ is a singleton $\} \subset Y$ is also residual.

Observe that each non-periodic doubly minimal system is not only minimal but also weakly mixing, and so totally minimal. In fact, let $(X, T)$ be a minimal weakly mixing t.d.s. and $m \in \mathbb{N}$. It is well known that the system $\left(X, T^{m}\right)$ is weakly mixing and each point of $\left(X, T^{m}\right)$ is minimal; hence $\left(X, T^{m}\right)$ is a minimal t.d.s. For each pair $x \neq y \in Y_{1}$, if $y=S_{1}^{n} x$ for some $n \neq 0$, then $\left(x, S_{1}^{-n} y\right)=(x, x)$ is proximal; and if $y \notin\left\{S_{1}^{i} x\right\}_{i=-\infty}^{\infty}$ (which implies that $S_{1}^{n} y \notin\left\{S_{1}^{i} x\right\}_{i=-\infty}^{\infty}$ for any $n \in \mathbb{Z})$, then $\left(x, S_{1}^{n} y\right)$ has a dense orbit and hence is also proximal for any $n \in \mathbb{Z}$. Thus $\left(Y_{1}, S_{1}\right)$, as a non-periodic doubly minimal system, is a POD system. For a given $n \in \mathbb{N}$, since $\left(Y_{1}, S_{1}\right) \times\left(Y_{1}, S_{1}^{2}\right) \times \cdots \times\left(Y_{1}, S_{1}^{n}\right)$ is minimal (by Proposition 3.3) it follows that $(Y, S) \times\left(Y, S^{2}\right) \times \cdots \times\left(Y, S^{n}\right)$ is also minimal [1, Proposition 2.5] (disjointness is preserved by an almost 1-1 extension [1, Theorem 2.6]). This implies that $\prod_{i=1}^{\infty}\left(Y, S^{i}\right)$ is minimal, as minimality is preserved by the inverse limit. Then by Proposition 3.2 , we get the conclusion.

Remark 3.6. In [39] the author showed that if each pair in a t.d.s. $(X, T)$ is positively recurrent under $T \times T$, then the entropy is zero. This is not true if we replace $T \times T$ by $T \times T^{2}$ by the proof of the previous proposition. Note that the question whether recurrence under $T \times T$ implies zero entropy is still open.

3.3. A related result. Finally, we shall present a result related to the property of hereditary lowerability. First, let's make some preparations (for details see [7, 17, 29, 31]).

Let $\pi:(X, T) \rightarrow(Y, S)$ be a factor map between t.d.s.s. The relative topological entropy of $(X, T)$ w.r.t. $\pi$ is defined as follows:

$$
h_{\mathrm{top}}(T, X \mid \pi)=\sup _{y \in Y} h\left(T, \pi^{-1}(y)\right)=\sup _{y \in Y} h^{B}\left(T, \pi^{-1}(y)\right) .
$$

Observe that (they are proved respectively as [17, Theorem 7.3], [11, Theorem 3.3] and [31, Theorem 4.2])

Proposition 3.7. Let $\pi:(X, T) \rightarrow(Y, S)$ be a factor map between t.d.s.s.

(1) If $E \subseteq X$ is compact, then

$$
h(S, \pi(E)) \leq h(T, E) \leq h(S, \pi(E))+h_{t o p}(T, X \mid \pi) .
$$

(2) If $K \subseteq X$, then

$$
h^{B}(S, \pi(K)) \leq h^{B}(T, K) \leq h^{B}(S, \pi(K))+h_{\text {top }}(T, X \mid \pi) .
$$

Let $(X, T)$ be a t.d.s. and $\mathcal{U}_{1}, \mathcal{U}_{2} \in \mathcal{C}_{X}^{o}$. Put $N\left(\mathcal{U}_{1} \mid \mathcal{U}_{2}\right)=\max \left\{N\left(\mathcal{U}_{1}, U_{2}\right): U_{2} \in\right.$ $\left.\mathcal{U}_{2}\right\}$. Then, for each $m, n \in \mathbb{N}$,

$$
\begin{aligned}
N\left(\left(\mathcal{U}_{1}\right)_{0}^{m+n-1} \mid\left(\mathcal{U}_{2}\right)_{0}^{m+n-1}\right) & \leq N\left(\left(\mathcal{U}_{1}\right)_{0}^{n-1} \mid\left(\mathcal{U}_{2}\right)_{0}^{m+n-1}\right) N\left(\left(\mathcal{U}_{1}\right)_{n}^{m+n-1} \mid\left(\mathcal{U}_{2}\right)_{0}^{m+n-1}\right) \\
& \leq N\left(\left(\mathcal{U}_{1}\right)_{0}^{n-1} \mid\left(\mathcal{U}_{2}\right)_{0}^{n-1}\right) N\left(\left(\mathcal{U}_{1}\right)_{n}^{m+n-1} \mid\left(\mathcal{U}_{2}\right)_{n}^{m+n-1}\right) \\
& =N\left(\left(\mathcal{U}_{1}\right)_{0}^{n-1} \mid\left(\mathcal{U}_{2}\right)_{0}^{n-1}\right) N\left(\left(\mathcal{U}_{1}\right)_{0}^{m-1} \mid\left(\mathcal{U}_{2}\right)_{0}^{m-1}\right),
\end{aligned}
$$

i.e. $\left\{\log N\left(\left(\mathcal{U}_{1}\right)_{0}^{n-1} \mid\left(\mathcal{U}_{2}\right)_{0}^{n-1}\right): n \in \mathbb{N}\right\}$ is sub-additive, and so we may set

$h\left(T, \mathcal{U}_{1} \mid \mathcal{U}_{2}\right)=\lim _{n \rightarrow+\infty} \frac{1}{n} \log N\left(\left(\mathcal{U}_{1}\right)_{0}^{n-1} \mid\left(\mathcal{U}_{2}\right)_{0}^{n-1}\right) \quad\left(=\inf _{n \in \mathbb{N}} \frac{1}{n} \log N\left(\left(\mathcal{U}_{1}\right)_{0}^{n-1} \mid\left(\mathcal{U}_{2}\right)_{0}^{n-1}\right)\right)$. 
Define the topologically conditional $\mathcal{U}_{2}$-entropy of $(X, T)$ by

$$
h^{*}\left(T, X \mid \mathcal{U}_{2}\right)=\sup _{\mathcal{U}_{1} \in \mathcal{C}_{X}^{o}} h\left(T, \mathcal{U}_{1} \mid \mathcal{U}_{2}\right)
$$

and the topologically conditional entropy of $(X, T)$ by

$$
h^{*}(T, X)=\inf _{\mathcal{U}_{2} \in \mathcal{C}_{X}^{o}} h^{*}\left(T, X \mid \mathcal{U}_{2}\right) .
$$

In particular, $h^{*}(T, X) \leq h_{\text {top }}(T, X)$. Observe that if $(X, T)$ is zero-dimensional, then by a standard construction we can represent $(X, T)$ as an inverse limit of sub-shifts over finite alphabets:

$$
(X, T)=\lim _{(}\left(X_{r}, T_{r}\right),
$$

where $X_{r} \subseteq \Lambda_{r}^{\mathbb{Z}}$ with $\Lambda_{r}$ a finite discrete space, $T_{r}$ is the left shift over $\Lambda_{r}^{\mathbb{Z}}$ and $\left(X_{r}, T_{r}\right)$ is a factor of $\left(X_{r+1}, T_{r+1}\right)$ for each $r \in \mathbb{N}$. Now let $\phi_{r}:(X, T) \rightarrow\left(X_{r}, T_{r}\right)$ be the natural homomorphism and $\mathcal{U}_{r}$ the clopen generating partition of $X_{r}(r \in \mathbb{N})$. Observe that the sequence $\left\{h_{\mathrm{top}}\left(T, X \mid \phi_{r}\right): r \in \mathbb{N}\right\}$ decreases. Here are some easy but useful facts:

(1) $h_{\text {top }}\left(T_{r}, X_{r}\right)=h_{\phi_{r}^{-1}\left(\mathcal{U}_{r}\right)}(T, X)$ and $h_{\text {top }}(T, X)=\lim _{r \rightarrow+\infty} h_{\text {top }}\left(T_{r}, X_{r}\right)$,

(2) $h_{\mathrm{top}}\left(T, X \mid \phi_{r}\right) \leq h^{*}\left(T, X \mid \phi_{r}^{-1}\left(\mathcal{U}_{r}\right)\right)$ and

(3) $h^{*}(T, X)=\lim _{r \rightarrow+\infty} h^{*}\left(T, X \mid \phi_{r}^{-1}\left(\mathcal{U}_{r}\right)\right) \geq \lim _{r \rightarrow+\infty} h_{\text {top }}\left(T, X \mid \phi_{r}\right)$.

Thus we have the following interesting result.

Theorem 3.8. Let $(X, T)$ be a t.d.s. with finite entropy and $K \subseteq X$ compact. If $h(T, K)>h^{*}(T, X)$, then for each $0 \leq h \leq h(T, K)-h^{*}(T, X)$ one has

$$
\overline{\left\{h\left(T, K^{\prime}\right): K^{\prime} \subseteq K \text { is compact }\right\}} \cap\left[h, h+h^{*}(T, X)\right] \neq \emptyset .
$$

Proof. It's well known that each t.d.s. with finite entropy has a zero-dimensional principal extension [5, Proposition 7.8], i.e. an extension preserving entropy for each invariant measure; and if $\pi$ is a principal extension of a system with finite entropy, then $\pi$ preserves the topologically conditional entropy [22, Theorem 3] and has zero relative topological entropy by conditional variational principles 9 , Theorems 3 and 4]. Thus, using Proposition 3.7 (1) we may assume that $(X, T)$ is zero-dimensional.

Moreover, it makes no difference to say $h \in\left[0, h(T, K)-h^{*}(T, X)\right)$.

We represent $(X, T)$ by an inverse limit of sub-shifts over finite alphabets

$$
(X, T)=\lim _{\longleftarrow}\left(X_{r}, T_{r}\right),
$$

with $\phi_{r}:(X, T) \rightarrow\left(X_{r}, T_{r}\right)$ the natural homomorphism for each $r \in \mathbb{N}$. Then

$$
h<h(T, K)-h^{*}(T, X) \leq h(T, K)-\lim _{r \rightarrow+\infty} h_{\mathrm{top}}\left(T, X \mid \phi_{r}\right) \text { (using fact (3)). }
$$

For each $r \in \mathbb{N}$ we set $K_{r}=\phi_{r}(K)$, so $h(T, K)-h_{\text {top }}\left(T, X \mid \phi_{r}\right) \leq h\left(T_{r}, K_{r}\right) \leq$ $h(T, K)$ (using Proposition 3.7(1) again). Thus if $r \in \mathbb{N}$ is large enough, then there exists a compact $K_{r}^{h} \subseteq K_{r}$ with $h\left(T_{r}, K_{r}^{h}\right)=h$ (using Theorem [17, Theorem 5.4]). Last, put $K_{r}^{(h)}=\phi_{r}^{-1}\left(K_{r}^{h}\right) \cap K$. As $\phi_{r}\left(K_{r}^{(h)}\right)=K_{r}^{h}$, by Proposition 3.7 (1) one has

$$
h=h\left(T_{r}, K_{r}^{h}\right) \leq h\left(T, K_{r}^{(h)}\right) \leq h\left(T_{r}, K_{r}^{h}\right)+h_{\text {top }}\left(T, X \mid \phi_{r}\right)=h+h_{\text {top }}\left(T, X \mid \phi_{r}\right) .
$$

We can claim the conclusion by fact (3). 


\section{A positive answer to Questions 1 and 4}

In this section we shall give a positive answer to Questions 1 and 4

Let $(X, T)$ be a t.d.s. Denote by $\mathcal{M}(X)$ (resp. $\left.\mathcal{M}(X, T), \mathcal{M}^{e}(X, T)\right)$ the set of all Borel probability measures (resp. $T$-invariant Borel probability measures, ergodic $T$-invariant Borel probability measures) on $X$. All of them are equipped with the weak star topology. Denote by $\mathcal{B}_{X}$ the set of all Borel subsets of $X$.

Before proceeding, we restate [17, Lemma 4.3 (2)] as follows (for the detailed introduction of the (relative) measure-theoretic entropy and the disintegration of a measure over a sub- $\sigma$-algebra, see for example [17, §4]).

Lemma 4.1. Let $(X, T)$ be a t.d.s., $\mu \in \mathcal{M}^{e}(X, T)$ and $\mathcal{C} \subseteq \mathcal{B}_{\mu}$ a T-invariant sub- $\sigma$-algebra (i.e. $T^{-1} \mathcal{C}=\mathcal{C}$ in the sense of $\mu$ ). Here $\mathcal{B}_{\mu}$ is the completion of $\mathcal{B}_{X}$ under $\mu$. If $\mu=\int_{X} \mu_{x} d \mu(x)$ is the disintegration of $\mu$ over $\mathcal{C}$, then, for $\mu$-a.e. $x \in X$, fixing each $x$, for each $\epsilon \in(0,1)$ there exists a compact subset $Z_{x}(\epsilon)$ of $X$ such that $\mu_{x}\left(Z_{x}(\epsilon)\right) \geq 1-\epsilon$ and $h^{B}\left(T, Z_{x}(\epsilon)\right)=h\left(T, Z_{x}(\epsilon)\right)=h_{\mu}(T, X \mid \mathcal{C})$.

We also need to state a relative version of the well-known Sinai Theorem, which is essentially found in [32. It was made explicit in [33, Theorem 5] and [37] (for another treatment of it see [20]). Before stating it, we need to make some preparations.

Recall that a k-element distribution $\mathbf{I}$ is a probability vector $\left(I_{1}, \cdots, I_{k}\right)$, i.e. $I_{1}, \cdots, I_{k} \geq 0$ and $I_{1}+\cdots+I_{k}=1, k \in \mathbb{N}$. The entropy of it is defined by

$$
H(\mathbf{I})=\sum_{i=1}^{k}-I_{i} \log I_{i} .
$$

From now on, for a given t.d.s. $(X, T)$ and $\mu \in \mathcal{M}(X)$, each $\alpha \in \mathcal{P}_{X}$ is ordered and associated with a distribution $\operatorname{dist} \alpha$ (i.e. $\alpha=\left(A_{1}, \cdots, A_{k}\right)$ is equipped with a fixed order and in this case dist $\left.\alpha=\left(\mu\left(A_{1}\right), \cdots, \mu\left(A_{k}\right)\right)\right)$.

A relative version of the well-known Sinai Theorem is stated as follows.

Lemma 4.2. Let $(X, T)$ be a t.d.s., $\mu \in \mathcal{M}^{e}(X, T)$ and $\alpha \in \mathcal{P}_{X}, \gamma \in \mathcal{P}_{X}$ with $\alpha \subseteq \bigvee_{i=-\infty}^{+\infty} T^{-i} \gamma$ (in the sense of $\mu$ ). Then, for each $k$-element distribution $\mathbf{I}, k \in \mathbb{N}$ with $H(\mathbf{I}) \leq h_{\mu}(T, \gamma)-h_{\mu}(T, \alpha)$, there exists $\beta \in \mathcal{P}_{X}$ satisfying that

(1) $\beta \subseteq \bigvee_{i=-\infty}^{+\infty} T^{-i} \gamma$ and $\operatorname{dist} \beta=\mathbf{I}$;

(2) the partitions $T^{i} \beta, i \in \mathbb{Z}$, are independent (in the sense of $\mu$ ); that is, if $B_{i_{1}} \in T^{i_{1}} \beta, i_{1} \in \mathbb{Z}$ and $B_{i_{2}} \in T^{i_{2}} \beta, i_{2} \in \mathbb{Z}$ with $i_{1} \neq i_{2}$, then $\mu\left(B_{i_{1}} \cap B_{i_{2}}\right)=$ $\mu\left(B_{i_{1}}\right) \mu\left(B_{i_{2}}\right)$;

(3) $\bigvee_{i=-\infty}^{+\infty} T^{-i} \beta$ is independent of $\bigvee_{i=-\infty}^{+\infty} T^{-i} \alpha$ (in the sense of $\mu$ ).

Thus, we have

Proposition 4.3. Let $(X, T)$ be a t.d.s. and $\mu \in \mathcal{M}^{e}(X, T)$. Then, for each $0 \leq$ $h \leq h_{\mu}(T, X)$, there exists a T-invariant sub- $\sigma$-algebra $\mathcal{C} \subseteq \mathcal{B}_{\mu}$ (in the sense of $\mu$ ) with $h_{\mu}(T, X \mid \mathcal{C})=h$. Here $\mathcal{B}_{\mu}$ is the completion of $\mathcal{B}_{X}$ under $\mu$.

Proof. When $h=h_{\mu}(T, X)$, we may take $\mathcal{C}=\{\emptyset, X\}$, and so $h_{\mu}(T, X \mid \mathcal{C})=h$.

Now we assume that $0 \leq h<h_{\mu}(T, X)$. It is not hard to see that we can take $\alpha \in \mathcal{P}_{X}$ with $h_{\mu}(T, \alpha)=h$. Moreover, by the Rokhlin Theorem about countable generators [35, 10.13] there exists a countable measurable partition $\gamma=$ $\left\{C_{1}, C_{2}, \cdots\right\}$ (i.e. there exists a sequence of partitions $\left\{\alpha_{n}: n \in \mathbb{N}\right\} \subseteq \mathcal{P}_{X}$ with 
$\left.\gamma=\alpha_{1} \vee \alpha_{2} \vee \cdots \doteq\left\{A_{1} \cap A_{2} \cap \cdots: A_{n} \in \alpha_{n}, n \in \mathbb{N}\right\}\right)$ where $\bigvee_{i=-\infty}^{+\infty} T^{-i} \gamma=\mathcal{B}_{\mu}$ in the sense of $\mu$.

Set $\eta_{0}=\{X\}, \gamma_{0}=\eta_{0} \vee \alpha$ and $\eta_{n}=\left\{C_{1}, C_{2}, \cdots, C_{n}, X \backslash \bigcup_{i=1}^{n} C_{i}\right\}, \gamma_{n}=\eta_{n} \vee$ $\alpha, n \in \mathbb{N}$. Then, for each $n \in \mathbb{N}$, by Lemma 4.2, there exists $\beta_{n} \in \mathcal{P}_{X}$ with $\beta_{n} \subseteq \bigvee_{i=-\infty}^{+\infty} T^{-i} \gamma_{n}$ (in the sense of $\mu$ ) such that

(1) the partitions $T^{i} \beta_{n}, i \in \mathbb{Z}$ are independent (in the sense of $\mu$ ),

(2) $\bigvee_{i=-\infty}^{+\infty} T^{-i} \beta_{n}$ is independent of $\bigvee_{i=-\infty}^{+\infty} T^{-i} \gamma_{n-1}$ (in the sense of $\mu$ ) and

(3) $H\left(\operatorname{dist} \beta_{n}\right)=h_{\mu}\left(T, \gamma_{n}\right)-h_{\mu}\left(T, \gamma_{n-1}\right)$.

From (2), one has

$$
h_{\mu}\left(T, \gamma_{n-1} \mid \bigvee_{i=-\infty}^{+\infty} T^{-i} \beta_{n}\right)=h_{\mu}\left(T, \gamma_{n-1}\right) .
$$

Moreover, observe that $\gamma_{n} \vee \beta_{n} \subseteq \bigvee_{i=-\infty}^{+\infty} T^{-i} \gamma_{n}$ (in the sense of $\mu$ ). Using the relative Pinsker formula (see for example [15, Lemma 1.1] or [41, Theorem 3.3]) we have

$$
h_{\mu}\left(T, \gamma_{n} \vee \beta_{n}\right)=h_{\mu}\left(T, \gamma_{n}\right)
$$

and

$$
\begin{aligned}
& h_{\mu}\left(T, \gamma_{n} \mid \bigvee_{i=-\infty}^{+\infty} T^{-i} \beta_{n} \vee \bigvee_{i=-\infty}^{+\infty} T^{-i} \gamma_{n-1}\right) \\
= & h_{\mu}\left(T, \gamma_{n} \mid \bigvee_{i=-\infty}^{+\infty} T^{-i} \beta_{n}\right)-h_{\mu}\left(T, \gamma_{n-1} \mid \bigvee_{i=-\infty}^{+\infty} T^{-i} \beta_{n}\right)\left(\text { as } \gamma_{n} \vee \gamma_{n-1}=\gamma_{n}\right) \\
= & h_{\mu}\left(T, \gamma_{n} \mid \bigvee_{i=-\infty}^{+\infty} T^{-i} \beta_{n}\right)-h_{\mu}\left(T, \gamma_{n-1}\right)(\text { by (4.1) }) \\
= & \left(h_{\mu}\left(T, \gamma_{n} \vee \beta_{n}\right)-h_{\mu}\left(T, \beta_{n}\right)\right)-h_{\mu}\left(T, \gamma_{n-1}\right) \\
= & h_{\mu}\left(T, \gamma_{n}\right)-h_{\mu}\left(T, \gamma_{n-1}\right)-h_{\mu}\left(T, \beta_{n}\right)(\text { by (4.2) }) \\
(4.3)= & H\left(\operatorname{dist} \beta_{n}\right)-h_{\mu}\left(T, \beta_{n}\right)(\text { by }(3))=0(\text { by (1)). }
\end{aligned}
$$

Put $\mathcal{C}=\bigvee_{n=1}^{+\infty} \bigvee_{i=-\infty}^{+\infty} T^{-i} \beta_{n}$. Note that, for each $k \in \mathbb{N}$, in the sense of $\mu$, $\bigvee_{i=-\infty}^{+\infty} T^{-i} \beta_{k+1}$ is independent of $\bigvee_{i=-\infty}^{+\infty} T^{-i} \gamma_{k}$ and

$$
\bigvee_{j=1}^{k} \bigvee_{i=-\infty}^{+\infty} T^{-i} \beta_{j} \vee \bigvee_{i=-\infty}^{+\infty} T^{-i} \alpha \subseteq \bigvee_{j=1}^{k} \bigvee_{i=-\infty}^{+\infty} T^{-i} \gamma_{j}=\bigvee_{i=-\infty}^{+\infty} T^{-i} \gamma_{k}
$$

One has that $\bigvee_{i=-\infty}^{+\infty} T^{-i} \beta_{k+1}$ is independent of $\bigvee_{j=1}^{k} \bigvee_{i=-\infty}^{+\infty} T^{-i} \beta_{j} \vee \bigvee_{i=-\infty}^{+\infty} T^{-i} \alpha$ Combing this with the fact that $\bigvee_{i=-\infty}^{+\infty} T^{-i} \beta_{1}$ is independent of $\bigvee_{i=-\infty}^{+\infty} T^{-i} \alpha$ (in the sense of $\mu$ ), we have that $\bigvee_{j=1}^{k} \bigvee_{i=-\infty}^{+\infty} T^{-i} \beta_{j}$ is independent of $\bigvee_{i=-\infty}^{+\infty} T^{-i} \alpha$ (in the sense of $\mu$ ) for each $k \in \mathbb{N}$, and so $\mathcal{C}$ is independent of $\bigvee_{i=-\infty}^{+\infty} T^{-i} \alpha$ in the sense of $\mu$.

Finally, we claim that $\mathcal{C}$ is just the sub- $\sigma$-algebra we need. Obviously, $T^{-1} \mathcal{C}=\mathcal{C}$. Now we are going to show $h_{\mu}(T, X \mid \mathcal{C})=h$. On one hand,

$$
h_{\mu}(T, X \mid \mathcal{C}) \geq h_{\mu}(T, \alpha \mid \mathcal{C})=h_{\mu}(T, \alpha)=h,
$$


where the last identity follows from the fact that $\mathcal{C}$ is independent of $\bigvee_{i=-\infty}^{+\infty} T^{-i} \alpha$. On the other hand, for each $n \in \mathbb{N}$, by the relative Pinsker formula

$$
\begin{aligned}
h_{\mu}\left(T, \gamma_{n} \mid \mathcal{C}\right)= & h_{\mu}(T, \alpha \mid \mathcal{C})+h_{\mu}\left(T, \eta_{n} \mid \mathcal{C} \vee \bigvee_{i=-\infty}^{+\infty} T^{-i} \alpha\right) \\
\leq & h+h_{\mu}\left(T, \eta_{n} \mid \bigvee_{j=1}^{n} \bigvee_{i=-\infty}^{+\infty} T^{-i} \beta_{j} \vee \bigvee_{i=-\infty}^{+\infty} T^{-i} \alpha\right) \\
= & h+h_{\mu}\left(T, \eta_{n} \mid \bigvee_{j=1}^{n} \bigvee_{i=-\infty}^{+\infty} T^{-i} \beta_{j} \vee \bigvee_{i=-\infty}^{+\infty} T^{-i} \alpha \vee \bigvee_{i=-\infty}^{+\infty} T^{-i} \eta_{n-1}\right) \\
& +h_{\mu}\left(T, \eta_{n-1} \mid \bigvee_{j=1}^{n} \bigvee_{i=-\infty}^{+\infty} T^{-i} \beta_{j} \vee \bigvee_{i=-\infty}^{+\infty} T^{-i} \alpha\right)\left(\operatorname{as} \eta_{n} \vee \eta_{n-1}=\eta_{n}\right) .
\end{aligned}
$$

By (4.3)

$$
\begin{aligned}
h_{\mu}\left(T, \gamma_{n} \mid \mathcal{C}\right) & \leq h+h_{\mu}\left(T, \eta_{n-1} \mid \bigvee_{j=1}^{n} \bigvee_{i=-\infty}^{+\infty} T^{-i} \beta_{j} \vee \bigvee_{i=-\infty}^{+\infty} T^{-i} \alpha\right) \\
& \leq h+h_{\mu}\left(T, \eta_{n-1} \mid \bigvee_{j=1}^{n-1} \bigvee_{i=-\infty}^{+\infty} T^{-i} \beta_{j} \vee \bigvee_{i=-\infty}^{+\infty} T^{-i} \alpha\right) \\
& \leq \cdots \leq h+h_{\mu}\left(T, \eta_{1} \mid \bigvee_{i=-\infty}^{+\infty} T^{-i} \beta_{1} \vee \bigvee_{i=-\infty}^{+\infty} T^{-i} \alpha\right)=h,
\end{aligned}
$$

which implies

$$
h_{\mu}(T, X \mid \mathcal{C})=\lim _{n \rightarrow+\infty} h_{\mu}\left(T, \bigvee_{i=-n}^{n} T^{-i} \gamma_{n} \mid \mathcal{C}\right)=\lim _{n \rightarrow+\infty} h_{\mu}\left(T, \gamma_{n} \mid \mathcal{C}\right) \leq h
$$

(as $\gamma_{1} \preceq \gamma_{2} \preceq \cdots$ and $\bigvee_{n \in \mathbb{N}} \bigvee_{i=-n}^{n} T^{-i} \gamma_{n}=\mathcal{B}_{\mu}$ (in the sense of $\mu$ )), and so $h_{\mu}(T, X \mid \mathcal{C})=h$. This ends the proof of the proposition.

Now we can answer Questions 1 and 4 affirmatively.

Theorem 4.4. Each t.d.s. $(X, T)$ is not only lowerable but also D-lowerable. In fact, for each $0 \leq h \leq h(T, X)$ there exists a compact $K_{h} \subseteq X$ with $h\left(T, K_{h}\right)=$ $h^{B}\left(T, K_{h}\right)=h$.

Proof. When $h=h(T, X)$, we may take $K_{h}=X$. When $h<h(T, X)$, by the classical variational principle (see for example [38, Corollary 8.6.1]) we may take $\mu \in \mathcal{M}^{e}(X, T)$ with $h_{\mu}(T, X) \geq h$. Then by Proposition 4.3 there exists a $T$ invariant sub- $\sigma$-algebra $\mathcal{C} \subseteq \mathcal{B}_{\mu}$ with $h_{\mu}(T, X \mid \mathcal{C})=h$, where $\mathcal{B}_{\mu}$ is the completion of $\mathcal{B}_{X}$ under $\mu$, and so there exists compact $K_{h} \subseteq X$ with $h\left(T, K_{h}\right)=h^{B}\left(T, K_{h}\right)=$ $h_{\mu}(T, X \mid \mathcal{C})=h$ (using Lemma 4.1).

Remark 4.5. We should remark that in 36] Shub and Weiss presented a t.d.s. with infinite entropy such that its each non-trivial factor has infinite entropy. 


\section{A partial answer to Question 5}

In this section, we shall give a partial answer to Question 5 by proving that each asymptotically $h$-expansive (equivalently, hereditarily uniformly lowerable) t.d.s. is D-hereditarily lowerable.

5.1. Each asymptotically $h$-expansive t.d.s. is D-hereditarily lowerable. Recall that, for a given t.d.s. $(X, T)$ with a compatible metric $d,(X, T)$ is called asymptotically $h$-expansive if $\lim _{\epsilon \rightarrow 0+} h_{T}^{*}(\epsilon)=0$, where $\epsilon>0$ and

$$
h_{T}^{*}(\epsilon)=\sup _{x \in X} h\left(T, \Phi_{\epsilon}(x)\right), \text { with } \Phi_{\epsilon}(x)=\left\{y \in X: d\left(T^{n} x, T^{n} y\right) \leq \epsilon \text { if } n \geq 0\right\} .
$$

Observe that it holds that $h^{*}(T, X)=\lim _{\epsilon \rightarrow 0+} h_{T}^{*}(\epsilon)([6, \S 4])$.

Lemma 5.1. Let $(X, T)$ be a symbolic t.d.s. Then $(X, T)$ is not only hereditarily lowerable but also D-hereditarily lowerable.

Proof. Note that any asymptotically $h$-expansive t.d.s. is hereditarily lowerable ([17, Theorem 7.7]), and so $(X, T)$ is hereditarily lowerable.

Now let $(X, T)$ be a subshift of $\left(\{1,2, \cdots, m\}^{\mathbb{Z}}, \sigma\right)$, where $m \geq 2$ and $\sigma$ is the left shift on $\{1,2, \cdots, m\}^{\mathbb{Z}}$. Let $\pi:\{1,2, \cdots, m\}^{\mathbb{Z}} \rightarrow \mathbb{T}$ with

$$
\pi\left(\left(x_{j}\right)_{j \in \mathbb{Z}}\right)=e^{2 \pi i\left(\sum_{j=0}^{+\infty} \frac{x_{j}-1}{m^{j+1}}\right)}
$$

for $\left(x_{j}\right)_{j \in \mathbb{Z}} \in\{1,2, \cdots, m\}^{\mathbb{Z}}$. Then $\pi:\left(\{1,2, \cdots, m\}^{\mathbb{Z}}, \sigma\right) \rightarrow\left(\mathbb{T}, T_{m}\right)$ is a factor map, where $T_{m}$ is defined by $T_{m}(z)=z^{m}$ for $z \in \mathbb{T}$.

For each $z \in \mathbb{T}$, there are $y^{1}, y^{2} \in\{1,2, \cdots, m\}^{\mathbb{Z}_{+}}$such that

$$
\pi^{-1}(z)=\left\{x=\left(x_{j}\right)_{j \in \mathbb{Z}} \in\{1,2, \cdots, m\}^{\mathbb{Z}}:\left(x_{j}\right)_{j=0}^{+\infty}=y^{1} \text { or } y^{2}\right\} ;
$$

here in fact for almost all $z \in \mathbb{T}, y^{1}=y^{2}$. Thus $h^{B}\left(T, \pi^{-1}(z)\right) \leq h\left(T, \pi^{-1}(z)\right)=0$, and so $h_{\text {top }}\left(\sigma,\{1,2, \cdots, m\}^{\mathbb{Z}} \mid \pi\right)=0$. Hence by Proposition 3.7

$$
h^{B}(\sigma, K)=h^{B}\left(\pi(K), T_{m}\right)
$$

for any $K \subseteq\{1,2, \cdots, m\}^{\mathbb{Z}}$. Since any continuous image of a Souslin set in $\{1,2, \cdots, m\}^{\mathbb{Z}}$ is Souslin, we know that $\left(\{1,2, \cdots, m\}^{\mathbb{Z}}, \sigma\right)$ is D-hereditarily lowerable by Proposition 2.5 (2) and (5.2). As a subsystem of $\left(\{1,2, \cdots, m\}^{\mathbb{Z}}, \sigma\right)$, $(X, T)$ is also D-hereditarily lowerable.

Theorem 5.2. Each asymptotically h-expansive t.d.s. is D-hereditarily lowerable.

Proof. Observe that each asymptotically $h$-expansive t.d.s. admits a principal extension to a symbolic t.d.s. ([5, Theorem 8.6]). As commented in the first paragraph of the proof of Theorem 3.8, a principal extension of a system with finite entropy has zero relative topological entropy, and so by Proposition 3.7 and Lemma 5.1 we obtain the conclusion.

Remark that, by the same argument presented in the proof of Theorem 3.8, we can obtain the following result with the help of Proposition 3.7 (2) and Lemma 5.1.

Proposition 5.3. Let $(X, T)$ be a t.d.s. with finite entropy and $K \subseteq X$ Souslin. If $h^{B}(T, K)>h^{*}(T, X)$, then for each $0 \leq h \leq h^{B}(T, K)-h^{*}(T, X)$ one has

$$
\overline{\left\{h^{B}\left(T, K^{\prime}\right): K^{\prime} \subseteq K\right\}} \cap\left[h, h+h^{*}(T, X)\right] \neq \emptyset .
$$

The property of asymptotical $h$-expansiveness can be weakened respectively as follows. 
Definition 5.4. Let $(X, T)$ be a t.d.s. with a compatible metric $d$. We call it

(1) anti-asymptotically $h$-expansive, if for each $\epsilon>0$ there exists a factor map $\pi:(Y, S) \rightarrow(X, T)$, where $(Y, S)$ is a symbolic t.d.s., such that $h_{\text {top }}(S, Y \mid \pi)<\epsilon$

(2) quasi-asymptotically $h$-expansive, if $\lim _{\epsilon \rightarrow 0+} h\left(T, \Phi_{\epsilon}(x)\right)=0$ for each $x \in$ $X$.

We remark that there is a D-hereditarily lowerable t.d.s. which is not quasiasymptotically $h$-expansive. Let $\left(X_{1}, T_{1}\right)$ be the full shift over $\Sigma_{2}=\{0,1\}^{\mathbb{Z}}$ and $\left(X_{2}, T_{2}\right)$ the identity transformation of the one point compactification over $\mathbb{Z}$ with $X_{2}=\mathbb{Z} \cup\{\infty\}$. Put $(Y, S)=\left(X_{1}, T_{1}\right) \times\left(X_{2}, T_{2}\right)$. Collapsing $X_{1} \times\{\infty\}$ to one point $x_{0}$ we get a t.d.s. $(X, T)$. It is not hard to check that $\lim _{\epsilon \rightarrow 0+} h\left(T, \Phi_{\epsilon}\left(x_{0}\right)\right)=$ $\log 2$, which implies that the t.d.s. $(X, T)$ is not quasi-asymptotically $h$-expansive, whereas, clearly $(X, T)$ is D-hereditarily lowerable by Theorem 5.2 .

In fact, we can construct a D-hereditarily lowerable t.d.s. which is not asymptotically $h$-expansive but quasi-asymptotically $h$-expansive.

Example 5.5. There is a D-hereditarily lowerable t.d.s. which is not asymptotically $h$-expansive but quasi-asymptotically $h$-expansive.

Proof. For each $n \in \mathbb{N}$ we choose $\epsilon_{n}>0$ and $C_{n} \subseteq[0,1]$ homeomorphic to the Cantor set with $\lim _{n \rightarrow+\infty} \epsilon_{n}=0$ and $\lim _{n \rightarrow+\infty} C_{n}=[0,1]$ (in the sense of Hausdorff metric). Set $X=X_{0} \cup X_{1} \cup X_{2} \cup \cdots$, where $X_{0}=\{0\} \times[0,1] \subseteq \mathbb{R}^{2}$ and $X_{n}=$ $\left\{\frac{1}{n}\right\} \times C_{n} \subseteq \mathbb{R}^{2}$ for each $n \in \mathbb{N}$. Now for each $n \in \mathbb{N}$ we let $T_{n}: X_{n} \rightarrow X_{n}$ be a minimal sub-shift such that $h\left(T_{n}, \Phi_{\epsilon_{n}}\left(x_{n}\right)\right) \geq \log 2$ for some $x_{n} \in X_{n}$ (we may assume that $\lim _{n \rightarrow+\infty} x_{n}=x_{0}$ ) and let $T_{0}: X_{0} \rightarrow X_{0}$ be the identity map. Last, $(X, T)$ is defined naturally. We may add the assumptions on the defined $\left(X_{n}, T_{n}\right), n \in \mathbb{N}$, such that $(X, T)$ forms a t.d.s.

We claim that the t.d.s. $(X, T)$ is the system we need. It is not hard to check that $(X, T)$ is a D-hereditarily lowerable t.d.s. Clearly, $\lim _{\epsilon \rightarrow 0+} h\left(T, \Phi_{\epsilon}(x)\right)=0$ for each $x \in X$ and so $(X, T)$ is quasi-asymptotically $h$-expansive, whereas,

$$
\lim _{\epsilon \rightarrow 0+} \sup _{x \in X} h\left(T, \Phi_{\epsilon}(x)\right) \geq \limsup _{n \rightarrow+\infty} h\left(T_{n}, \Phi_{\epsilon_{n}}\left(x_{n}\right)\right) \geq \log 2,
$$

that is, t.d.s. $(X, T)$ is not asymptotically $h$-expansive.

To finish our example it remains to construct a t.d.s. $\left(X_{n}, T_{n}\right)$ and $\epsilon_{n}>0$ as above for each $n \in \mathbb{N}$. Let $n \in \mathbb{N}$. For each $j=1, \cdots, 2 n$ we put $C_{n}^{j}=\frac{2 j}{4 n+1}+C_{n}^{0} \subseteq$ $J_{n}^{j}=\left[\frac{2 j}{4 n+1}, \frac{2 j+1}{4 n+1}\right]$ with $C_{n}^{0}$ being linearly homeomorphic to the standard Cantor set and set $C_{n}=\bigcup_{j=0}^{2 n} C_{n}^{j}$. Define a permutation $\phi_{n}:\{0,1, \cdots, 2 n\} \rightarrow\{0,1, \cdots, 2 n\}$ such that $\phi_{n}^{j}(i)=i, 1 \leq j \leq 2 n+1$ implies $j=2 n+1$ and $\left|\phi_{n}(i)-i\right| \leq 2$ for each $0 \leq i \leq 2 n$. For example, we set $\phi_{n}(0)=2, \phi_{n}(2)=4, \cdots, \phi_{n}(2 n-2)=$ $2 n, \phi_{n}(2 n)=2 n-1 ; \phi_{n}(2 n-1)=2 n-3, \phi_{n}(2 n-3)=2 n-5, \cdots, \phi_{n}(3)=1$ and $\phi_{n}(1)=0$. Now let $\left(C_{n}^{0}, S_{n}\right)$ be a minimal sub-shift with $h\left(S_{n}, C_{n}^{0}\right) \geq(2 n+$ 1) $\log 2$. We define $T_{n}: X_{n} \rightarrow X_{n}$ as follows: $T_{n}\left(\frac{1}{n}, y_{n}\right)=\left(\frac{1}{n}, \frac{2 \phi_{n}(j)}{4 n+1}+c_{n}\right)$ if $y_{n} \notin C_{n}^{\phi_{n}^{-1}(0)}$ with $y_{n}=\frac{2 j}{4 n+1}+c_{n}$, where $c_{n} \in C_{n}^{0}$, and $T_{n}\left(\frac{1}{n}, y_{n}\right)=\left(\frac{1}{n}, S_{n}\left(c_{n}\right)\right)$ if $y_{n}=\frac{2 \phi_{n}^{-1}(0)}{4 n+1}+c_{n} \in C_{n}^{\phi_{n}^{-1}(0)}$, where $c_{n} \in C_{n}^{0}$. Let $\epsilon_{n}=\frac{5}{4 n+1}$. It is easy to check that the constructed $\left(X_{n}, T_{n}\right)$ is a minimal sub-shift. Moreover, for each $x_{n} \in C_{n}^{0}$, 
$C_{n}^{0} \subseteq \Phi_{\epsilon_{n}}\left(x_{n}\right)$, and so (by Lemma $3.1(1)$ )

$$
\begin{aligned}
h\left(T_{n}, \Phi_{\epsilon_{n}}\left(x_{n}\right)\right) & =\frac{1}{2 n+1} h\left(T_{n}^{2 n+1}, \Phi_{\epsilon_{n}}\left(x_{n}\right)\right) \\
& \geq \frac{1}{2 n+1} h\left(T_{n}^{2 n+1}, C_{n}^{0}\right)=\frac{1}{2 n+1} h\left(S_{n}, C_{n}^{0}\right) \geq \log 2
\end{aligned}
$$

(in fact, this works for each $x_{n} \in X_{n}$ ). Last, by the assumption of $\phi_{n}$ it is not hard to check that the defined $(X, T)$ is a t.d.s. This finishes the construction of our example.

Remark 5.6. In the above example we can choose $\left(C_{n}^{0}, S_{n}\right), n \in \mathbb{N}$, such that $h_{\text {top }}(T, X)=+\infty$, and so one has that there is a D-hereditarily lowerable t.d.s. (with entropy infinite) which is not anti-asymptotically $h$-expansive but quasiasymptotically $h$-expansive. We do not know if there is such an example which is minimal.

5.2. Each anti-asymptotically $h$-expansive t.d.s. is asymptotically $h$-expansive. In this subsection we will show that asymptotical $h$-expansiveness and anti-asymptotical $h$-expansiveness are equivalent properties. For that, we need some notions and results in 5 .

Given a t.d.s. $(X, T)$, we will say a sequence of partitions $\left\{\alpha_{k}\right\}$ of $X$ is refining if the maximum diameter of elements of $\alpha_{k}$ goes to zero with $k$, and for each $k$ the partition $\alpha_{k+1}$ refines $\alpha_{k}$. The partitions have small boundaries if their boundaries have measure zero for all $\mu$ in $\mathcal{M}(X, T)$. For a finite entropy t.d.s. $(X, T)$ admitting a non-periodic minimal factor, by [25, Theorem 6.2] and [26, Theorem 4.2] we know that $(X, T)$ has the so-called small boundary property, which is equivalent to the existence of a basis of the topology consisting of sets whose boundaries have measure zero for every invariant measure. Moreover, it is easy to construct the refining sequence of partitions with small boundaries for $(X, T)$ (see [5, Theorem $7.6(3)])$.

Definition 5.7. Let $(X, T)$ be a finite entropy t.d.s. admitting a non-periodic minimal factor. An entropy structure for $(X, T)$ is a sequence $\mathcal{H}$ of functions $\left\{h_{k}\right\}$ defined on $\mathcal{M}(X, T)$ in the following way: suppose $\left\{\alpha_{k}\right\}$ is a refining sequence of finite Borel partitions with small boundaries; then $h_{k}: \mathcal{M}(X, T) \rightarrow \mathbb{R}$ is obtained by setting $\mu \mapsto h_{\mu}\left(T, \alpha_{k}\right)$ for each $k \in \mathbb{N}$.

The following result follows from [5, Theorem 8.6].

Proposition 5.8. Let $(X, T)$ be a finite entropy t.d.s. admitting a non-periodic minimal factor. The following statements are equivalent for $(X, T)$ with entropy structure $\mathcal{H}$ :

(1) $(X, T)$ is asymptotically h-expansive.

(2) $h_{k}$ converges uniformly to the entropy function $h$, where $h(\mu):=h_{\mu}(T, X)$ for each $\mu \in \mathcal{M}(X, T)$.

Definition 5.9. A function $f: K \rightarrow \mathbb{R}$ defined on a compact metric space $K$ is upper semicontinuous (u.s.c.) if one of the following equivalent conditions holds:

(1) $f=\inf _{i \in I} f_{i}$ for some family $\left\{f_{i}\right\}_{i \in I}$ of continuous functions.

(2) $f=\lim _{i \rightarrow+\infty} g_{i}$, where $\left\{g_{i}\right\}$ is a non-increasing sequence of continuous functions. 
(3) For each $r \in \mathbb{R}$, the set $\{x \in K: f(x) \geq r\}$ is closed.

(4) $\limsup _{x^{\prime} \rightarrow x} f\left(x^{\prime}\right) \leq f(x)$ at each $x \in K$.

For u.s.c functions, the following properties hold:

a) The infimum of any family of u.s.c. functions is u.s.c. (by Definition 5.9 $(1))$.

b) Both the sum and the supremum of finitely many u.s.c. functions are u.s.c. (by Definition 5.9 (2)).

c) Every u.s.c. function from a compact metric space to $\mathbb{R}$ is bounded above and attains its maximum.

The following result is [5, Proposition 2.4].

Proposition 5.10 (Exchanging suprema and infima). If $\left\{f_{i}\right\}_{i \in \mathbb{N}}$ is a non-increasing sequence of u.s.c. functions on a compact metric space $K$, then

$$
\inf _{i \in \mathbb{N}} \sup _{x \in K} f_{i}(x)=\sup _{x \in K} \inf _{i \in \mathbb{N}} f_{i}(x) .
$$

Theorem 5.11. Asymptotical h-expansiveness and anti-asymptotical $h$-expansiveness are equivalent properties.

Proof. Remark that each asymptotically $h$-expansive t.d.s. admits a principal extension to a symbolic t.d.s. which has zero relative topological entropy (cf. the proof of Theorem 5.2). Thus an asymptotically $h$-expansive t.d.s. is clearly antiasymptotically $h$-expansive by definitions.

Conversely, let $(X, T)$ be an anti-asymptotically $h$-expansive t.d.s. Suppose $(Z, R)$ is an aperiodic minimal zero entropy system. Let $Y=X \times Z$ and $S=T \times R$. Then $(Y, S)$ is an anti-asymptotically $h$-expansive t.d.s., since $(Z, R)$ is asymptotically $h$-expansive.

Now $(Y, S)$ is a finite entropy t.d.s. admitting a non-periodic minimal factor $(Z, R)$. Suppose $\left\{\alpha_{k}\right\}$ is a refining sequence of finite Borel partitions of $(Y, S)$ with small boundaries. Define $\mathcal{H}$ by setting $h_{k}: \mu \mapsto h_{\mu}\left(S, \alpha_{k}\right)$ for each $k \in \mathbb{N}$. Then $\mathcal{H}$ is an entropy structure of $(Y, S)$.

For $m \in \mathbb{N}$, let $\pi_{m}:\left(X_{m}, T_{m}\right) \rightarrow(Y, S)$ be a factor map such that $\left(X_{m}, T_{m}\right)$ is a symbolic t.d.s. and $h_{\text {top }}\left(T_{m}, X_{m} \mid \pi_{m}\right)<\frac{1}{m}$. Then $\pi_{m}$ induces a continuous map $\pi_{m}^{*}: \mathcal{M}\left(X_{m}, T_{m}\right) \rightarrow \mathcal{M}(Y, S)$ satisfying $\pi_{m}^{*} \nu(A)=\nu\left(\pi_{m}^{-1} A\right)$ for any $\nu \in$ $\mathcal{M}\left(X_{m}, T_{m}\right)$ and any Borel subset $A$ of $Y$.

Let $\beta_{m}$ be a generating clopen partition of $\left(X_{m}, T_{m}\right)$. Now we consider the function $g_{k}^{m}: \mathcal{M}\left(X_{m}, T_{m}\right) \rightarrow \mathbb{R}$ with $g_{k}^{m}(\nu)=h_{\nu}\left(T_{m}, X_{m}\right)-h_{\pi_{m}^{*} \nu}\left(S, \alpha_{k}\right)$ for each $\nu \in \mathcal{M}\left(X_{m}, T_{m}\right)$. Then for $k \in \mathbb{N}$,

$$
\begin{aligned}
g_{k}^{m}(\nu) & =h_{\nu}\left(T_{m}, \beta_{m} \vee \pi_{m}^{-1}\left(\alpha_{k}\right)\right)-h_{\nu}\left(T_{m}, \pi_{m}^{-1} \alpha_{k}\right) \\
& =\lim _{N \rightarrow+\infty} \frac{1}{N} H_{\nu}\left(\bigvee_{i=0}^{N-1} T_{m}^{-i}\left(\beta_{m} \vee \pi_{m}^{-1}\left(\alpha_{k}\right)\right) \mid \bigvee_{i=0}^{N-1} T_{m}^{-i} \pi_{m}^{-1} \alpha_{k}\right) \\
& =\inf _{N \geq 1} \frac{1}{N} H_{\nu}\left(\bigvee_{i=0}^{N-1} T_{m}^{-i}\left(\beta_{m} \vee \pi_{m}^{-1}\left(\alpha_{k}\right)\right) \mid \bigvee_{i=0}^{N-1} T_{m}^{-i} \pi_{m}^{-1} \alpha_{k}\right) .
\end{aligned}
$$

The last equality follows from the fact that the sequence

$$
a_{n}(\nu):=H_{\nu}\left(\bigvee_{i=0}^{n-1} T_{m}^{-i}\left(\beta_{m} \vee \pi_{m}^{-1}\left(\alpha_{k}\right)\right) \mid \bigvee_{i=0}^{n-1} T_{m}^{-i} \pi_{m}^{-1} \alpha_{k}\right)
$$


is subadditive, i.e. $a_{n_{1}+n_{2}}(\nu) \leq a_{n_{1}}(\nu)+a_{n_{2}}(\nu)$. Since $\beta_{m}$ and $\pi_{m}^{-1} \alpha_{k}$ have small boundaries, $\nu \mapsto H_{\nu}\left(\bigvee_{i=0}^{N-1} T_{m}^{-i}\left(\beta_{m} \vee \pi_{m}^{-1}\left(\alpha_{k}\right)\right) \mid \bigvee_{i=0}^{N-1} T_{m}^{-i} \pi_{m}^{-1} \alpha_{k}\right)$ is a continuous function on $\mathcal{M}\left(X_{m}, T_{m}\right)$ for each $N \in \mathbb{N}$. Thus the function $g_{k}^{m}$ is u.s.c. by Definition 5.9 (1).

Next, we let $f_{k}^{m}: \mathcal{M}(Y, S) \rightarrow \mathbb{R}$ with

$$
\begin{aligned}
f_{k}^{m}(\mu): & =\sup \left\{g_{k}^{m}(\nu): \nu \in\left(\pi_{m}^{*}\right)^{-1}(\mu)\right\} \\
& =\sup \left\{h_{\nu}\left(T_{m}, X_{m}\right): \nu \in\left(\pi_{m}^{*}\right)^{-1}(\mu)\right\}-h_{\mu}\left(S, \alpha_{k}\right)
\end{aligned}
$$

for each $\mu \in \mathcal{M}(Y, S)$. Since $g_{k}^{m}$ is u.s.c., it is easily seen using Definition 5.9 (4) that $f_{k}^{m}$ is also u.s.c.

Now let $f_{k}(\mu):=h_{\mu}(S, Y)-h_{\mu}\left(S, \alpha_{k}\right)$ for each $\mu \in \mathcal{M}(Y, S)$. Then $\left\{f_{k}\right\}$ is a decreasing sequence of non-negative functions on $\mathcal{M}(Y, S)$ and $\lim _{k \rightarrow+\infty} f_{k}(\mu)=0$ for all $\mu \in \mathcal{M}(Y, S)$. Since

$$
\begin{aligned}
f_{k}^{m}(\mu)-f_{k}(\mu) & =\sup \left\{h_{\nu}\left(T_{m}, X_{m}\right)-h_{\pi_{m}^{*} \nu}(S, Y): \nu \in\left(\pi_{m}^{*}\right)^{-1}(\mu)\right\} \\
& =\sup \left\{h_{\nu}\left(T_{m}, X_{m} \mid \pi_{m}\right): \nu \in\left(\pi_{m}^{*}\right)^{-1}(\mu)\right\} \\
& \in\left[0, h_{\text {top }}\left(T_{m}, X_{m} \mid \pi_{m}\right)\right] \subseteq\left[0, \frac{1}{m}\right]
\end{aligned}
$$

for any $\mu \in \mathcal{M}(Y, S)$, we have $f_{k}=\inf _{m \in \mathbb{N}} f_{k}^{m}$. Thus $f_{k}$ is u.s.c. since each $f_{k}^{m}$ is u.s.c.

By Proposition 5.10

$$
\lim _{k \rightarrow+\infty} \sup _{\mu \in \mathcal{M}(Y, S)} f_{k}(\mu)=\inf _{k \in \mathbb{N}} \sup _{\mu \in \mathcal{M}(Y, S)} f_{k}(\mu)=\sup _{\mu \in \mathcal{M}(Y, S)} \inf _{k \in \mathbb{N}} f_{k}(\mu)=0 .
$$

Thus $h_{k}$ converges uniformly to the entropy function $h$, where $h_{k}(\mu)=h_{\mu}\left(S, \alpha_{k}\right)$ and $h(\mu)=h_{\mu}(S, Y)$. Hence the system $(Y, S)$ is asymptotically $h$-expansive by Proposition 5.8 .

Finally since for any $x \in X, z \in Z$ and $\epsilon>0, \Phi_{\epsilon}((x, z)) \supset \Phi_{\epsilon}(x) \times\{z\}$ by definition (5.1), we have $h_{T \times R}^{*}(\epsilon) \geq h_{T}^{*}(\epsilon)$. So $\lim _{\epsilon \rightarrow 0+} h_{T}^{*}(\epsilon)=0$ since $\lim _{\epsilon \rightarrow 0+} h_{T \times R}^{*}(\epsilon)$ $=0$. Thus $(X, T)$ is asymptotically $h$-expansive.

\section{ACKNOWLEDGEMENT}

The authors would like to thank Downarowicz, Glasner and Weiss for useful discussions. The authors would also like to thank the referee for the careful reading and useful comments that resulted in substantial improvements to this paper.

\section{REFERENCES}

[1] Ethan Akin and Eli Glasner, Residual properties and almost equicontinuity, J. Anal. Math. 84 (2001), 243-286, DOI 10.1007/BF02788112. MR1849204 (2002f:37020)

[2] Rufus Bowen, Entropy for group endomorphisms and homogeneous spaces, Trans. Amer. Math. Soc. 153 (1971), 401-414. MR0274707 (43 \#469)

[3] Rufus Bowen, Entropy-expansive maps, Trans. Amer. Math. Soc. 164 (1972), 323-331. MR0285689 (44 \#2907)

[4] Rufus Bowen, Topological entropy for noncompact sets, Trans. Amer. Math. Soc. 184 (1973), 125-136. MR0338317 (49 \#3082)

[5] Mike Boyle and Tomasz Downarowicz, The entropy theory of symbolic extensions, Invent. Math. 156 (2004), no. 1, 119-161, DOI 10.1007/s00222-003-0335-2. MR2047659 (2005d:37015) 
[6] Mike Boyle, Doris Fiebig, and Ulf Fiebig, Residual entropy, conditional entropy and subshift covers, Forum Math. 14 (2002), no. 5, 713-757, DOI 10.1515/form.2002.031. MR 1924775 (2003g:37024)

[7] Tomasz Downarowicz, Entropy of a symbolic extension of a dynamical system, Ergodic Theory Dynam. Systems 21 (2001), no. 4, 1051-1070, DOI 10.1017/S014338570100150X. MR.1849601 (2002e:37016)

[8] T. Downarowicz and Y. Lacroix, Almost 1-1 extensions of Furstenberg-Weiss type and applications to Toeplitz flows, Studia Math. 130 (1998), no. 2, 149-170. MR.1623344(2000a:28014)

[9] Tomasz Downarowicz and Jacek Serafin, Fiber entropy and conditional variational principles in compact non-metrizable spaces, Fund. Math. 172 (2002), no. 3, 217-247, DOI 10.4064/fm172-3-2. MR 1898686 (2003b:37027)

[10] Kenneth Falconer, Fractal geometry, John Wiley \& Sons Ltd., Chichester, 1990. Mathematical foundations and applications. MR.1102677 (92j:28008)

[11] Chun Fang, Wen Huang, Yingfei Yi, and Pengfei Zhang, Dimensions of stable sets and scrambled sets in positive finite entropy systems, Ergodic Theory Dynam. Systems 32 (2012), no. 2, 599-628, DOI 10.1017/S0143385710000982. MR2901362

[12] Harry Furstenberg, Disjointness in ergodic theory, minimal sets, and a problem in Diophantine approximation, Math. Systems Theory 1 (1967), 1-49. MR0213508 (35 \#4369)

[13] H. Furstenberg, Recurrence in ergodic theory and combinatorial number theory, Princeton University Press, Princeton, N.J., 1981. M. B. Porter Lectures. MR603625 (82j:28010)

[14] Eli Glasner, Ergodic theory via joinings, Mathematical Surveys and Monographs, vol. 101, American Mathematical Society, Providence, RI, 2003. MR.1958753 (2004c:37011)

[15] E. Glasner, J.-P. Thouvenot, and B. Weiss, Entropy theory without a past, Ergodic Theory Dynam. Systems 20 (2000), no. 5, 1355-1370, DOI 10.1017/S0143385700000730. MR.1786718 (2001h:37011)

[16] Wen Huang, Xiangdong Ye, and Guohua Zhang, A local variational principle for conditional entropy, Ergodic Theory Dynam. Systems 26 (2006), no. 1, 219-245, DOI 10.1017/S014338570500043X. MR2201946 (2006j:37015)

[17] Wen Huang, Xiangdong Ye, and Guohua Zhang, Lowering topological entropy over subsets, Ergodic Theory Dynam. Systems 30 (2010), no. 1, 181-209, DOI 10.1017/S0143385709000066. MR2586351 (2011f:37027)

[18] Jonathan L. King, A map with topological minimal self-joinings in the sense of del Junco, Ergodic Theory Dynam. Systems 10 (1990), no. 4, 745-761, DOI 10.1017/S0143385700005873. MR.1091424 (92a:54036)

[19] A. Katok, Lyapunov exponents, entropy and periodic orbits for diffeomorphisms, Inst. Hautes Études Sci. Publ. Math. 51 (1980), 137-173. MR.573822 (81i:28022)

[20] John C. Kieffer, A simple development of the Thouvenot relative isomorphism theory, Ann. Probab. 12 (1984), no. 1, 204-211. MR723739 (85g:28025)

[21] H. B. Keynes and D. Newton, Real prime flows, Trans. Amer. Math. Soc. 217 (1976), 237255. MR0400189(53\#4024)

[22] F. Ledrappier, A variational principle for the topological conditional entropy, Ergodic theory (Proc. Conf., Math. Forschungsinst., Oberwolfach, 1978), Lecture Notes in Math., vol. 729, Springer, Berlin, 1979, pp. 78-88. MR550412 (80j:54011)

[23] François Ledrappier and Peter Walters, A relativised variational principle for continuous transformations, J. London Math. Soc. (2) 16 (1977), no. 3, 568-576. MR0476995 (57 \#16540)

[24] Elon Lindenstrauss, Lowering topological entropy, J. Anal. Math. 67 (1995), 231-267, DOI 10.1007/BF02787792. MR1383496 (97b:54029)

[25] Elon Lindenstrauss, Mean dimension, small entropy factors and an embedding theorem, Inst. Hautes Études Sci. Publ. Math. 89 (1999), 227-262 (2000). MR1793417(2001j:37033)

[26] Elon Lindenstrauss and Benjamin Weiss, Mean topological dimension, Israel J. Math. 115 (2000), 1-24, DOI 10.1007/BF02810577. MR1749670 (2000m:37018)

[27] Pertti Mattila, Geometry of sets and measures in Euclidean spaces, Cambridge Studies in Advanced Mathematics, vol. 44, Cambridge University Press, Cambridge, 1995. Fractals and rectifiability. MR 1333890 (96h:28006)

[28] M. Misiurewicz, Diffeomorphism without any measure with maximal entropy (English, with Russian summary), Bull. Acad. Polon. Sci. Sér. Sci. Math. Astronom. Phys. 21 (1973), 903910. MR0336764(49\#1537) 
[29] Michał Misiurewicz, Topological conditional entropy, Studia Math. 55 (1976), no. 2, 175-200. MR0415587(54 \#3672)

[30] Michał Misiurewicz, On Bowen's definition of topological entropy, Discrete Contin. Dyn. Syst. 10 (2004), no. 3, 827-833, DOI 10.3934/dcds.2004.10.827. MR2018882 (2004i:37029)

[31] Piotr Oprocha and Guohua Zhang, Dimensional entropy over sets and fibres, Nonlinearity 24 (2011), no. 8, 2325-2346, DOI 10.1088/0951-7715/24/8/009. MR.2819452 (2012f:37032)

[32] Donald Ornstein, Two Bernoulli shifts with infinite entropy are isomorphic, Advances in Math. 5 (1970), 339-348. MR.0274716 (43 \#478a)

[33] Donald S. Ornstein and Benjamin Weiss, Unilateral codings of Bernoulli systems, Israel J. Math. 21 (1975), no. 2-3, 159-166. Conference on Ergodic Theory and Topological Dynamics (Kibbutz Lavi, 1974). MR0412386 (54 \#512)

[34] Yakov B. Pesin, Dimension theory in dynamical systems, Chicago Lectures in Mathematics, University of Chicago Press, Chicago, IL, 1997. Contemporary views and applications. MR:1489237(99b:58003)

[35] V. A. Rohlin, Lectures on the entropy theory of transformations with invariant measure (Russian), Uspehi Mat. Nauk 22 (1967), no. 5 (137), 3-56. MR0217258 (36 \#349)

[36] M. Shub and B. Weiss, Can one always lower topological entropy?, Ergodic Theory Dynam. Systems 11 (1991), no. 3, 535-546, DOI 10.1017/S0143385700006325. MR1125888 (92i:58105)

[37] Jean-Paul Thouvenot, Quelques propriétés des systèmes dynamiques qui se décomposent en un produit de deux systèmes dont l'un est un schéma de Bernoulli (French, with English summary), Israel J. Math. 21 (1975), no. 2-3, 177-207. Conference on Ergodic Theory and Topological Dynamics (Kibbutz, Lavi, 1974). MR0399419 (53 \#3263)

[38] Peter Walters, An introduction to ergodic theory, Graduate Texts in Mathematics, vol. 79, Springer-Verlag, New York, 1982. MR648108 (84e:28017)

[39] B. Weiss, Multiple recurrence and doubly minimal systems, Topological dynamics and applications (Minneapolis, MN, 1995), Contemp. Math., vol. 215, Amer. Math. Soc., Providence, RI, 1998, pp. 189-196, DOI 10.1090/conm/215/02940. MR1603185(99a:28018)

[40] Xiangdong Ye and Guohua Zhang, Entropy points and applications, Trans. Amer. Math. Soc. 359 (2007), no. 12, 6167-6186 (electronic), DOI 10.1090/S0002-9947-07-04357-7. MR2336322 (2008m:37026)

[41] Guohua Zhang, Relative entropy, asymptotic pairs and chaos, J. London Math. Soc. (2) 73 (2006), no. 1, 157-172, DOI 10.1112/S0024610705022520. MR2197376 (2006k:37035)

Wu Wen-Tsun Key Laboratory of Mathematics, UStC, Chinese Academy of Sciences and Department of Mathematics, University of Science and Technology of China, Hefei, Anhui, 230026, People's Republic of China

E-mail address: wenh@mail.ustc.edu.cn

Wu Wen-Tsun Key Laboratory of Mathematics, UstC, Chinese Academy of Sciences and Department of Mathematics, University of Science and Technology of China, Hefei, Anhui, 230026, People's Republic of China

E-mail address: yexd@ustc.edu.cn

School of Mathematical Sciences and LMnS, Fudan University, Shanghai 200433, CHINA

E-mail address: zhanggh@fudan.edu.cn 